\begin{tabular}{|c|c|c|c|c|c|c|}
\hline \multirow{4}{*}{ Impact Factor: } & ISRA (India) & $=3.117$ & SIS (USA) & $=0.912$ & ICV (Poland) & $=6.630$ \\
\hline & ISI (Dubai, UAE & $=0.829$ & РИНЦ (Russia) & $=0.156$ & PIF (India) & $=1.940$ \\
\hline & GIF (Australia) & $=0.564$ & ESJI (KZ) & $=\mathbf{5 . 0 1 5}$ & IBI (India) & $=4.260$ \\
\hline & JIF & $=1.500$ & SJIF (Morocco) & $=5.667$ & & \\
\hline
\end{tabular}

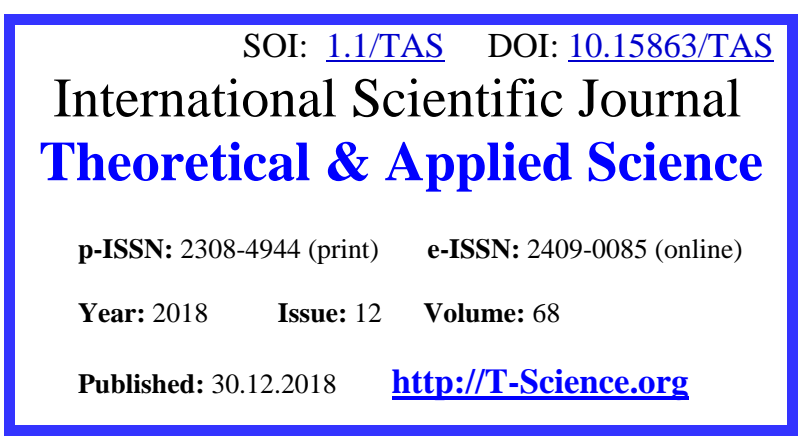

SECTION 7. Mechanics and machine construction.
QR - Issue
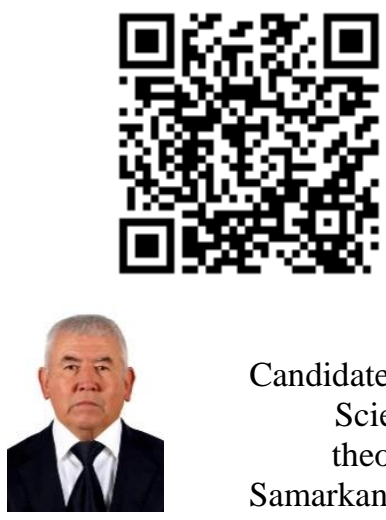

Ablakul Abdirashidov

Candidate of Physical and Mathematical Sciences, Docent to department of theoretical and applied mechanics,

Samarkand State University, Uzbekistan abdira@mail.ru

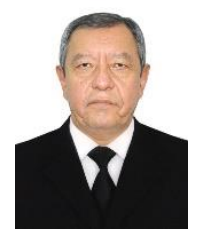

Abduvali Karshiyev

Candidate of Physical and Mathematical Sciences, Head of department of Computer engineering, Samarkand branch of Tashkent

University of Information Technologies, Samarkand, Uzbekistan,

\title{
DISTRIBUTION OF PRESSURE WAVES IN THE UNLIMITED WATER, THEIR REFLECTION FROM THE HARD SCREEN AND THE FREE SURFACE OF THE FLUID
}

Abstract: Numerical calculations are given in the axisymmetric formulation of the expansion of explosion products in infinite water, the formation of underwater waves, their reflection from the rigid screen and the free surface of the liquid. Also, pressure fields were calculated around an instantaneous detonating cylindrical charge varying in radius and thickness and located in an unlimited aqueous medium.

Key words: cylindrical charge; hard screen; free surface; underwater wave; pressure; bubble.

Language: Russian

Citation: Abdirashidov, A., \& Karshiyev, A. (2018). Distribution of pressure waves in the unlimited water, their reflection from the hard screen and the free surface of the fluid. ISJ Theoretical \& Applied Science, 12 (68), 316-322.

Soi: http://s-o-i.org/1.1/TAS-12-68-47 Doi: crossef https://dx.doi.org/10.15863/TAS.2018.12.68.47

\section{РАСПРОСТРАНЕНИЕ ВОЛН ДАВЛЕНИЯ В БЕЗГРАНИЧНОЙ ВОДЕ, ИХ ОТРАЖЕНИЕ ОТ ЖЕСТКОГО ЭКРАНА И СВОБОДНОЙ ПОВЕРХНОСТИ ЖИДКОСТИ}

Аннотация: Приведены численные расчеты в осесимметричной постановке расширения продуктов взрыва в безграничной воде, формирования подводных волн, их отражение от жесткого экрана и свободной поверхности жидкости. Также рассчитывали поля давления вокруг мгновенно детонирующего иุилиндрического заряда, изменяющегося по радиусу и толщчине и находящегося в безграничной водной среде.

Ключевые слова: ичилиндрический заряд; жесткий экран; свободная поверхность; подводная волна; давление; пузырь.

Введение.

В работе изучено воздействие заряда цилиндрической формы на окружающую среду (рис.1).

Поведение жидкости и газа описывалось уравнением сохранения в цилиндрической системе координат при $S_{i j}=0$, а их состояние - общеизвестным уравнением в форме Тэта и адиабатическим приближением соответственно $[2,4]$. Система уравнений гидродинамики дополнялась краевыми и начальными условиями: на поверхности контакта - условия равенства скоростей частиц сред, а на бесконечности условия покоя жидкости; начальные условия 


\begin{tabular}{|c|c|c|c|c|c|c|}
\hline \multirow{4}{*}{ Impact Factor: } & ISRA (India) & $=3.117$ & SIS (USA) & $=0.912$ & ICV (Poland) & $=6.630$ \\
\hline & ISI (Dubai, UAE & $=0.829$ & РИНЦ (Russia) & $=0.156$ & PIF (India) & $=1.940$ \\
\hline & GIF (Australia) & $=0.564$ & ESJI (KZ) & $=\mathbf{5 . 0 1 5}$ & IBI (India) & $=4.260$ \\
\hline & JIF & $=1.500$ & SJIF (Morocco) & $=5.667$ & & \\
\hline
\end{tabular}

полагались нулевыми (кроме давления в газе). Для совместного решения уравнений гидродинамики использовался численный метод М.Уилкинса $[2,4,7]$.

1. Распространение взрывной волны в безграничной водной среде.

Расчет проведен для небольшого цилиндрического заряда взрывчатого вещества, детонирующего в безграничной жидкости. Продукты детонации и вода предполагаются идеальными, невязкими и сжимаемыми.

В рассматриваемом случае задача изучается в следующей последовательности. Взрывчатое превращение ВВ и расширение продуктов детонации происходит по схеме мгновенной детонации. При детонации во всем объеме заряда мгновенно устанавливаются давление и плотность продуктов детонации, равная начальной плотности ВВ. После этого ударная волна генерируется в воде, а в области газа распространяется волна разрежения. Авторами известных работ [1-3] показано существование вторичной ударной волны. За волной разрежения следует вторая ударная волна, которая образуется у границы раздела, движется обратно в направлении к центру и после прихода в центр отражается. Достигнув границы раздела между газом и водой, ударная волна частично переходит в воду, а частично отражается. Процесс внутреннего отражения скачка и частичной передачи его давления в воду может многократно повторяться. Таким образом, течение внутри газового объема представляет собой последовательность волн разрежения, связанных с расширением, и последовательность вторичных волн сжатия, которые накладываются на волны разрежения.

Начальное состояние воды, окружающей заряд, было взято таким: $P_{0}=1$ атм; $\rho_{0}=1000$ кг $/ \mathrm{M}^{3}$, а для газового пузыря, образовавшегося

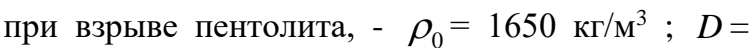
7655 м/с - скорость детонации; $P=0.125 \rho_{0} D^{2}$; толщина и диаметр заряда равны 0.02 м.

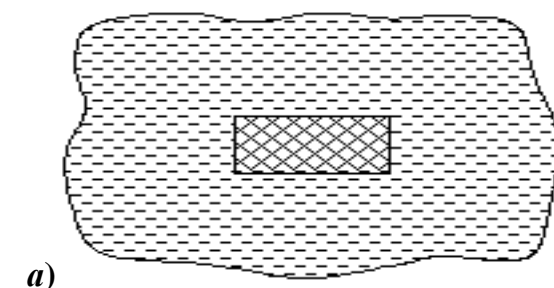

б)
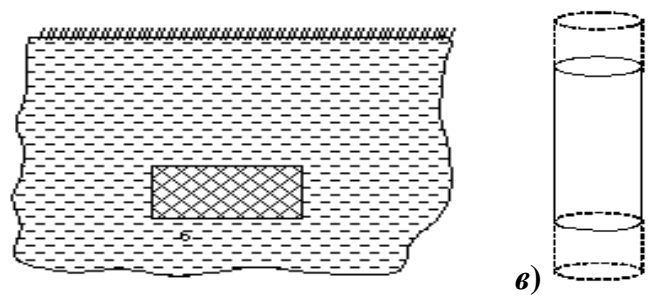

2)

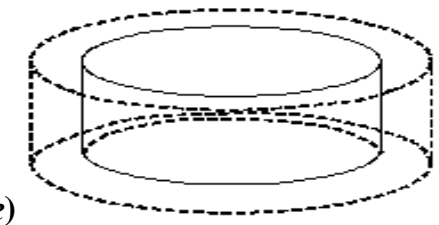

Рис. 1. Объект исследования: $a$ - взрыв в безграничной жидкости; б - взрыв вблизи твердой границы. в, 2 - виды цилиндрических зарядов в безграничной жидкости.

В расчетах использовалась сеточная область, состоящая из 50 х 50 ячеек, причем область, занятая взрывчатым веществом, разбивалась на квадратную сетку с шагом 0.2 см. Ширина ячеек, прилегающих к газовому объему, равнялась 0.35 см. Начальная ширина остальных ячеек в воде задавалась так, чтобы ширина каждой ячейки в 1.005 раз превосходила ширину предшествующей ячейки. Ячейки представляют собой цилиндрические кольца. Поскольку расчеты проводятся в лагранжевых переменных, то масса каждой ячейки фиксируется при начальном выборе положения границ ячеек и остается постоянной в продолжение всего расчета.

При расчетах использовался переменный временной шаг. В начале расчета он равнялся $\tau$ $=0.05$ мкс. Далее $\tau$ находилась на условиях устойчивости Куранта. Для этого в каждом шаге по времени определялась ячейка минимальных размеров по радиальному и осевому направлениям. Затем находим шаг по времени для жидкости и газа, обеспечивающих устойчивые численные расчеты. В расчетах используем минимальную из этих двух величин. Когда газовый пузырь расширяется до размеров объема газа, ширина ячеек жидкости, прилегающих к газовому объему, становится небольшой из-за радиального и осевого сжатия. Тогда эти размеры ячейки определяют наибольший шаг по времени, который может быть взят для расчетов. Для прослеживания за распространением ударной волны до расстояния, равного нескольким десяткам радиуса заряда, следует использовать последовательно алгоритм перестройки сетки в окружности газового объема. 


\begin{tabular}{|c|c|c|c|c|c|c|}
\hline \multirow{4}{*}{ Impact Factor: } & ISRA (India) & $=3.117$ & SIS (USA) & $=0.912$ & ICV (Poland) & $=6.630$ \\
\hline & ISI (Dubai, UAI & $=0.829$ & РИНЦ (Russia) & $=0.156$ & PIF (India) & $=1.940$ \\
\hline & GIF (Australia) & $=0.564$ & ESJI (KZ) & $=5.015$ & IBI (India) & $=4.260$ \\
\hline & JIF & $=1.500$ & SJIF (Morocco) & $=5.667$ & & \\
\hline
\end{tabular}

Расчеты заканчивались, когда основная ударная волна проходила от границы раздела газвода расстояние 12 р.з. (р.з. - радиус заряда). Время расчетов охватывало лишь небольшую часть полного периода колебания пузыря [4]. В конце расчета радиус пузыря и шаг по времени равнялись 3.7 р.з. и 0.02 мкс соответственно, а время составляло 70 мкс.

На рис. 2 приведен график относительных пиковых значений давления на ударной волне, построенный в зависимости от положения основного ударного фронта в воде. Эти значения представляют собой величины относительных пиковых давлений, взятых с кривых, связывающих давление и расстояние при фиксированном времени.

На рисунке штриховыми линиями обозначены расчетные данные, полученные для сферического пентолитного заряда. Этот взрыв описан в работе [6], и приведенные данные считаются надежными. Видно, что максимальные давления на фронте ударной волны мало отличаются. Пиковые давления на расстоянии больше 10 р.з. находились в соответствии с хорошими результатами работы [6]. Кроме того, спад пиковых давлений на фронте подводной волны происходит ближе по закону, установленному для сферических подводных волн. В окрестности газового пузыря давление на ударной волне резко падает. Например, когда ударный фронт находится на расстоянии 2.1 р.3., пиковое давление падает до $1100 \mathrm{MПа}$
Зависимость радиуса газового пузыря от времени показана на рис.3. Дана также расчетная кривая изменения радиуса сферического пузыря со временем (штриховая кривая), опубликованная Ч.Мейдером [6]. Видно, что согласие между кривыми удовлетворительное. Эти кривые характеризуют небольшую часть полного периода колебания радиуса пузыря.

Рассмотрим движение волны в жидкости. Зависимость давления от расстояния для момента времени $t=65$ мкс, когда подводная волна прошла расстояние 12 р.з., изображена на рис.4. В этом положении ударной волны давление в газовом пузыре не является равномерно распределенным и меняется от 0.78 МПа на границе раздела между газом и водой до 0.007 МПа в центре.

Так же рассчитывали поля давления вокруг мгновенно детонирующего цилиндрического заряда, изменяющегося по радиусу и толщине и находящегося в безграничной водной среде. Численные расчеты проводили по осесимметричной лагранжевой схеме с искусственной вязкостью для зарядов с отношениями длины к радиусу, равными $1,2,4,8$, и наоборот. В этом случае также использовали алгоритм расчета предыдущей задачи.

На рис.5,a,б приведена зависимость относительного давления на фронте подводной ударной волны от относительного радиуса и толщины при взрывах цилиндрических зарядов.

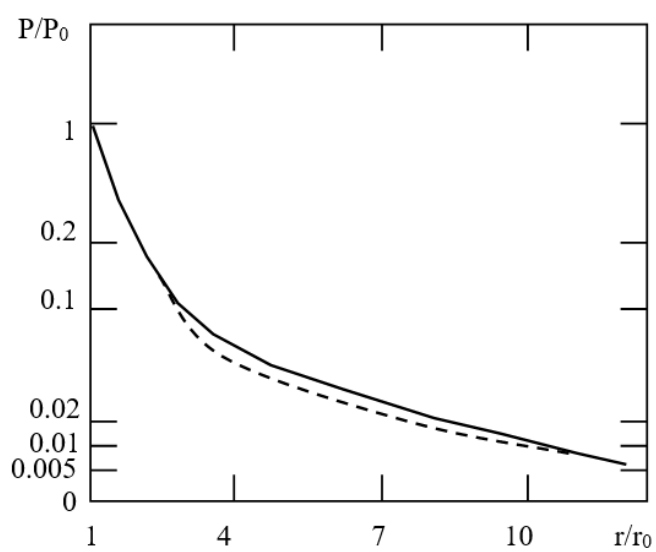

Рис.2. Зависимость относительных пиковых давлений на ударном фронте от относительного радиуса. 


\begin{tabular}{|c|c|c|c|c|c|c|}
\hline \multirow{4}{*}{ Impact Factor: } & ISRA (India) & $=3.117$ & SIS (USA) & $=0.912$ & ICV (Poland) & $=6.630$ \\
\hline & ISI (Dubai, UAE & $=0.829$ & РИНЦ (Russia & $=0.156$ & PIF (India) & $=1.940$ \\
\hline & GIF (Australia) & $=0.564$ & ESJI (KZ) & $=5.015$ & IBI (India) & $=4.260$ \\
\hline & JIF & $=1.500$ & S.JIF (Morocco & $=5.667$ & & \\
\hline
\end{tabular}

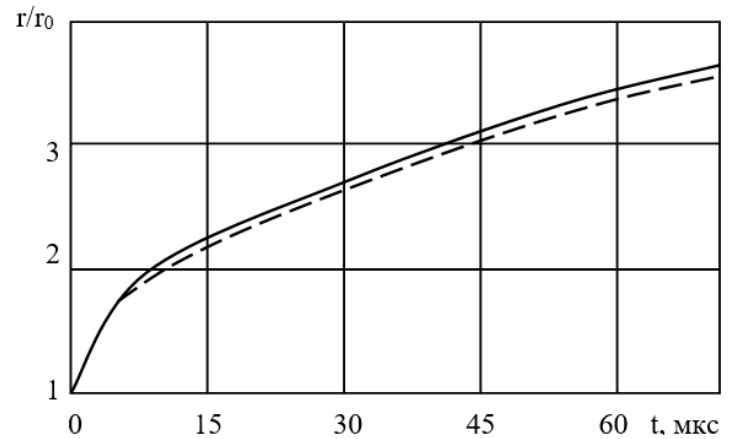

Рис.3. Относительный радиус границ раздела между продуктами детонации и водой, построенный как функция от времени для случая цилиндрического (сплошная линия) и сферического (штриховая линия) заряда.

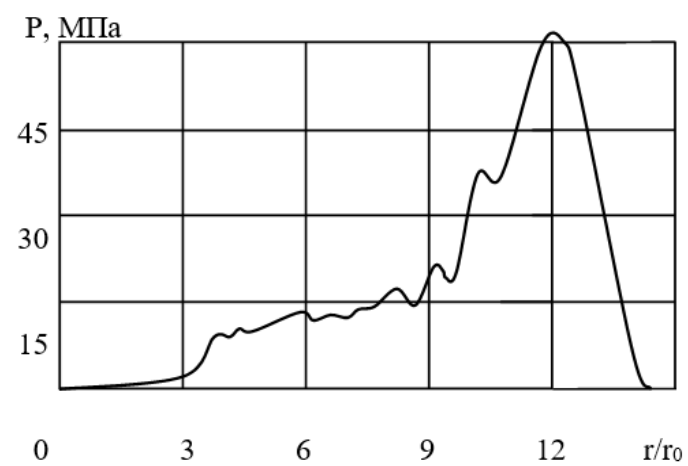

Рис.4. Давление, рассчитанное в зависимости от радиуса в момент времени $t=6.5 \cdot 10^{-5} c$.

Рис.6 иллюстрирует зависимость относительной толщины $y=h / h_{0}$ и радиуса $y=r / r_{0} \quad$ цилиндрического заряда от времени. Положение фронта ударной волны и газового пузыря определяли до относительных расстояний
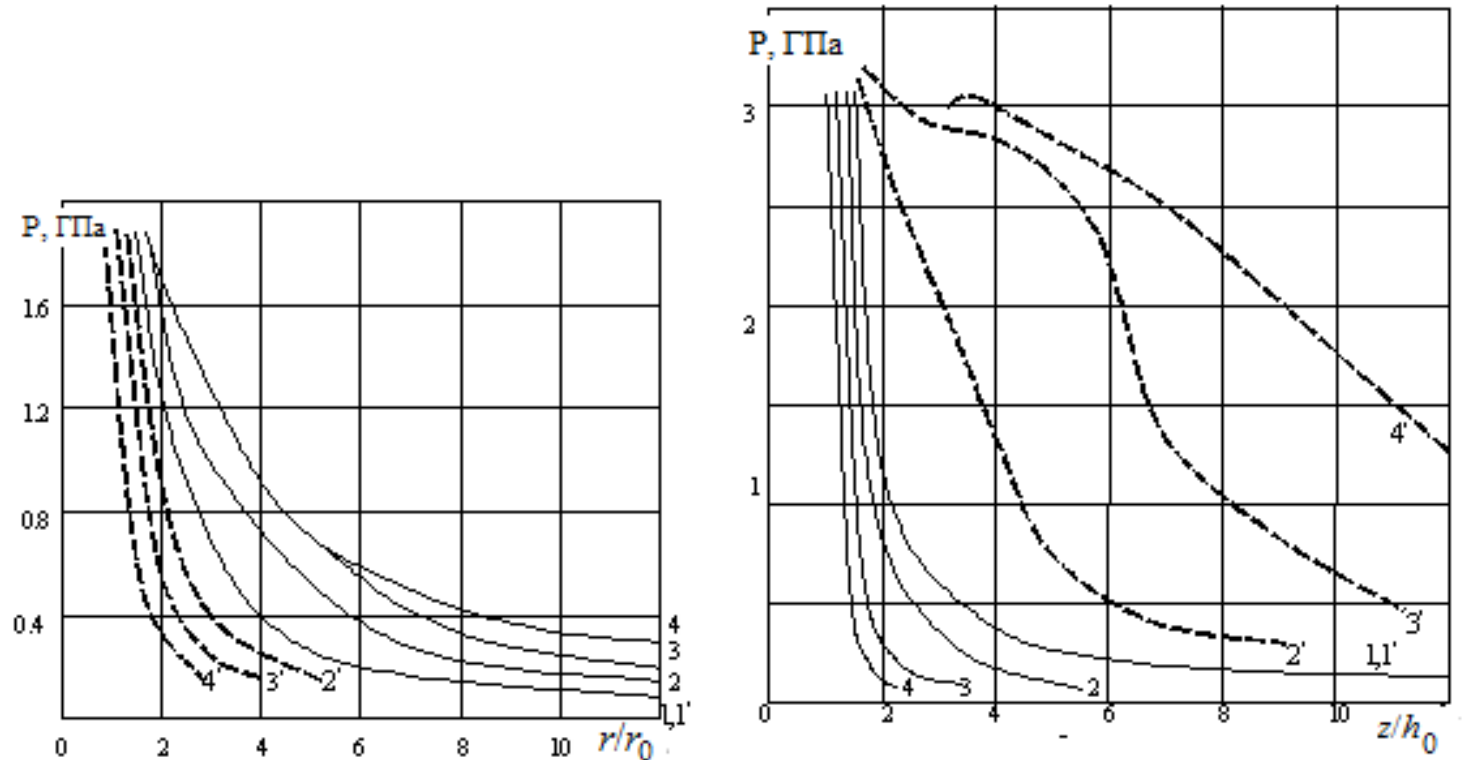

Рис.5. Зависимость относительного давления на фронте подводной ударной волны от относительного радиуса $(a)$ и относительной толщины ( $\sigma)$ при взрывах цилиндрических зарядов для $\mathbf{r}_{0}=1$ см и ho=1 см (I); 2 см (2); 4 см (3); 8 см (4) (сплошные линии) и для ho=1 и ro=1 см (I); 2 см (2); 4 см (3); 8 см (4) (штриховые линии). 


\begin{tabular}{|c|c|c|c|c|c|c|}
\hline \multirow{4}{*}{ Impact Factor: } & ISRA (India) & $=3.117$ & SIS (USA) & $=0.912$ & ICV (Poland) & $=6.630$ \\
\hline & ISI (Dubai, UAF & $=0.829$ & РИНЦ (Russia) & $=0.156$ & PIF (India) & $=1.940$ \\
\hline & GIF (Australia) & $=0.564$ & ESJI (KZ) & $=\mathbf{5 . 0 1 5}$ & IBI (India) & $=4.260$ \\
\hline & JIF & $=1.500$ & SJIF (Morocco) & $=5.667$ & & \\
\hline
\end{tabular}

На основе проведенных расчетов установлено, что спад давления на фронте ударной волны происходит по закону, установленному для сферических подводных волн; расширение газового пузыря и максимальное давление на фронте ударной волны по горизонтальному и вертикальному направлениям одинаково; в процессе отражения ударной волны от неподвижной границы, ее пиковое значение возрастает почти в два раза от значения перед отражением; с увеличением радиуса заряда максимальное давление на фронте ударной волны по радиусу падает с расстоянием быстрее, чем по оси симметрии; спад давления по закону, установленному для сферических волн, начинается с расстояния $r / r_{0}>2$ и $z / h_{0}>2$ при удлинении заряда и $r / r_{0}>2$ и $z / h_{0}>10$ при увеличении радиуса заряда.
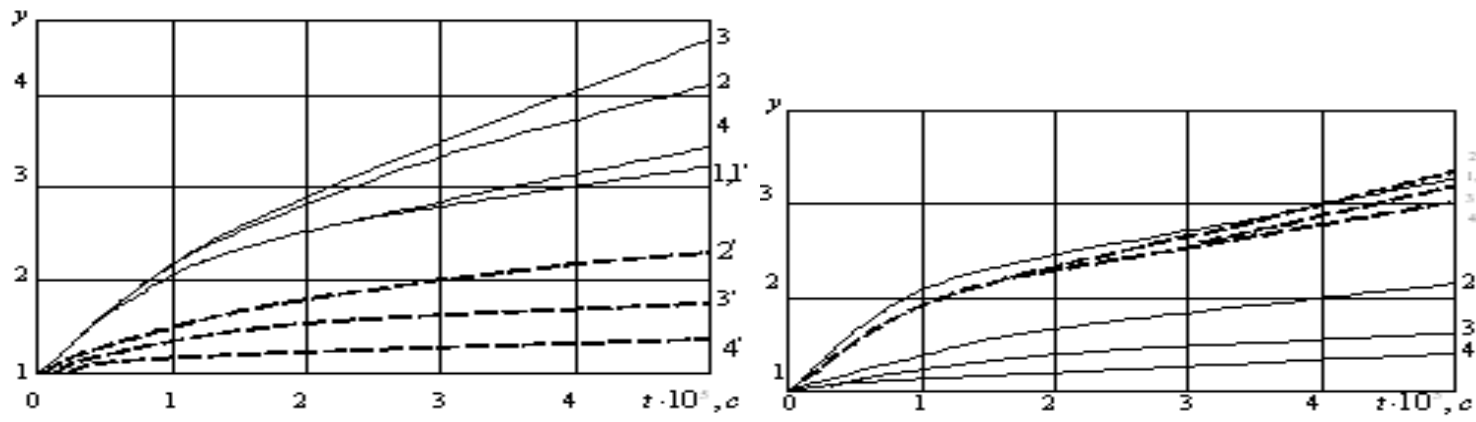

Рис.6. Зависимость относительной толщины $\mathbf{y}=\mathrm{h} / \mathrm{h}_{0}$ (сплошные кривые) и радиуса $\mathbf{y}=\mathbf{r} / \mathbf{r}_{0}$ (штриховые кривые) цилиндрического заряда от времени:

а) для $\mathbf{h}_{0}=\mathbf{1}$ см и $\mathbf{r}_{0}=\mathbf{1}$ см $\left(1,1^{\prime}\right) ; \mathbf{2}$ см $\left(2,2^{\prime}\right) ; \mathbf{4}$ см $\left(3,3^{\prime}\right) ; \mathbf{8}$ см $\left(4,4^{\prime}\right)$;

б) для $\mathbf{r}_{0}=\mathbf{1}$ см и $\mathbf{h}_{0}=\mathbf{1}$ см $\left(1,1^{\prime}\right) ; \mathbf{2}$ см $\left(2,2^{\prime}\right) ; \mathbf{4}$ см $\left(3,3^{\prime}\right) ; \mathbf{8}$ см $\left(4,4^{\prime}\right)$.

\section{2. Подводный взрыв вблизи свободной поверхности жидкости \\ Изучены волновые процессы, возникающие} при нестационарном расширении газового пузыря вблизи свободной поверхности жидкости. При относительно малых глубинах расположения источника волны возникают кумулятивные выемки, и эта задача сводится к расчету зоны разрушения жидкости [2, 4, 5]. Эксперименты показали, что при расширении сферического газового пузыря вблизи свободной поверхности после отражения ударной волны от сферической поверхности возникают кумулятивные выемки, зона разрушения, также развивается зона кавитации, которая настолько глубока, что даже охватывает газовый пузырь [2].

По алгоритму первой задачи проведено численное исследование влияние глубины погружения на зоны разрушения и кавитации в жидкости. Начальное давление в газовом пузыре равнялось 200 МПа, а погружение пузыря варьировалось $d=6 \quad \ldots 12$ см. Давление на свободной поверхности воды равнялось атмосферному.

Результаты расчета показали, что вначале вблизи свободной поверхности возникает зона разрушения, а потом формируется зона кавитации. При заглублении пузыря зона разрушения постепенно исчезает, а зона кавитации, становясь все более однородной, поднимается относительно пузыря, все более примыкая к свободной поверхности. Размеры зон разрушения и кавитации существенно зависят от начального давления в газовом пузыре и от величины статического давления на поверхность жидкости. Возникновение кавитации может существенно сказаться на характере выбросов жидкости и формировании нелинейных поверхностных волн.

\section{3. Подводный взрыв вблизи недеформи-} руемой стенки.

В качестве твердой границы рассматриваем фиксированную границу, ограничивающую полубесконечный объем жидкости. Вблизи этой границы происходит высокоскоростное расширение газового объема, возникающего после мгновенного подрыва пентолитого заряда цилиндрической формы. В этом случае используем вышеописанный алгоритм расчета динамики подводного взрыва. Расстояние между зарядом и стенкой равно 6 р.з. Некоторые профили давления для рассматриваемого случая показаны на рис.7. Направления движения подводной волны и волны отражения показаны стрелками, а граница раздела между газом и водой - вертикальными черточками; штриховыми линиями указаны величины пиковых давлений на ударном фронте. 


\begin{tabular}{|c|c|c|c|c|c|c|}
\hline \multirow{4}{*}{ Impact Factor: } & ISRA (India) & $=3.117$ & SIS (USA) & $=0.912$ & ICV (Poland) & $=6.630$ \\
\hline & ISI (Dubai, UAE & $=0.829$ & РИНЦ (Russia) & $=0.156$ & PIF (India) & $=1.940$ \\
\hline & GIF (Australia) & $=0.564$ & ESJI (KZ) & $=5.015$ & IBI (India) & $=4.260$ \\
\hline & JIF & $=1.500$ & SJIF (Morocco) & $=5.667$ & & \\
\hline
\end{tabular}

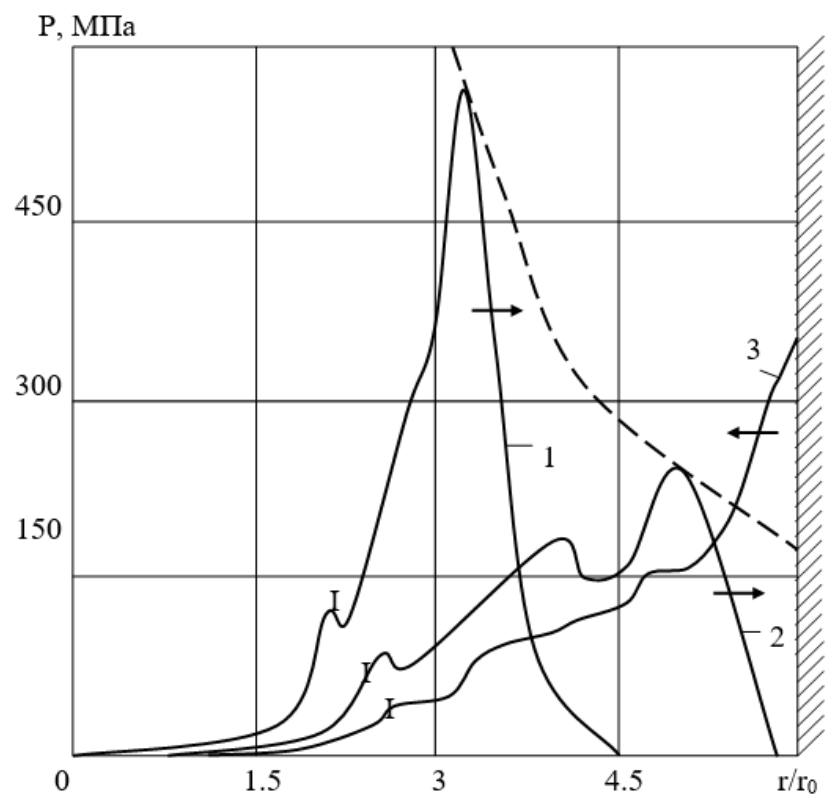

Рис.7. Давление в волнах отражающихся от жесткого экрана:

$$
1-t=10^{-5} c, 2-t=2 \cdot 10^{-5} c, 3-t=2.5 \cdot 10^{-5} c \text {. }
$$

Давление отраженной волны выше, чем давление от падающей ударной волны. Например, пиковое давление ударной волны на расстояние 5 р.з. равно 230 МПа $(t=20$ мкс), а давление отраженной волны поднимается до 350 МПа $(t=25$ мкс), т.е. пиковое значение волны давления приблизительно удваивается при отражении от жесткого экранах [2, 4]. В расчетах анализировали только первые мгновения отражения ударной волны.

\section{Выводы.}

Рассмотренный алгоритм и результаты работы можно использовать при решении многих задач гидроупругопластичности [7-11], т.е. при изучении нестационарного взаимодействия плит, пластин и оболочечных элементов конструкций с деформируемой средой (например, с жидкостью или грунтом) при импульсном и гидродинамическом нагружениях.

\section{References:}

1. Galiev, S. U. (1981). Dynamics of hydroelastoplastic system. (p.376). Kiev: Naukova Dumka publ.

2. Galiev, S. U. (1988). Nonlinear waves in the restricted continuous medium. (p.263). Kiev: Naukova Dumka publ..

3. Guz, A. N., Kubenko, V. D., \& Babaev, A. E. (2002). Dynamics of system of the shells immersed in a liquid. Appl. mech., 38, № 3, 260-301.

4. Khudoynazarov, K., \& Abdirashidov, A. (2005). Nonstationary interaction elastoplastic deformable elements of structures with fluid. (p.220). Tashkent: Fan publ..
5. Galiev, S. U., et al. (1989). Numerical Modelling of wave processes in restricted medium. (p.201). Kiev: Naukova Dumka Publ..

6. Mader C. L. (1979). Numerical modeling of detonations. University of California Press, Berkeley - Los Angeles - London 1979, first edition, 485.

7. Lepixin, P. P., Romashenko, V. A. (2014). Prochnost neodnorodnix anizotropnix polix silindrov pri impulsnom nagrujenii. (p.228). Kiyev: Nauk. dumka.

8. Shmakov, V. P. (2011). Izbranniye trudi po gidrouprugosti i dinamike uprugix konstruksiy. (p.288). Izd-vo MGTU im N.E.Baumana. 


\begin{tabular}{|c|c|c|c|c|c|c|}
\hline \multirow{4}{*}{ Impact Factor: } & ISRA (India) & $=3.117$ & SIS (USA) & $=0.912$ & ICV (Poland) & $=6.630$ \\
\hline & ISI (Dubai, UAF & $=0.829$ & РИНЦ (Russia) & $=0.156$ & PIF (India) & $=1.940$ \\
\hline & GIF (Australia) & $=0.564$ & ESJI (KZ) & $=\mathbf{5 . 0 1 5}$ & IBI (India) & $=4.260$ \\
\hline & JIF & $=1.500$ & SJIF (Morocco) & $=5.667$ & & \\
\hline
\end{tabular}

9. Panovko, Y. G. (2017). Mexanika deformiruyemogo tverdogo tela. Sovremenniye konsepsii, oshibki i paradoksi. (p.288). Izd-vo Lenand.

10. Veklich, N. (2014). Nestasionarniye zadachi gidrouprugosti dlya plastin.
Testoviye zadachi. (p.276). LAP LAMBERT Academic Publishing.

11. Ageyev, R. V., Popov, V. S., \& Mogilevich, L. I. (2012). Gidrouprugost trexsloynix plastin, vzaimodeystvuyushix so sloyem jidkosti. (p.112). LAP LAMBERT Academic Publishing. 